\title{
Stability via successive approximation for nonlinear implicit fractional differential equations
}

\author{
KISHOR D. KUCCHE ${ }^{a}$ AND SAgar T. SUtAR ${ }^{b}$
}

\begin{abstract}
In this paper we are concerned with nonlinear implicit fractional differential equations with initial conditions. We prove the existence and uniqueness results by using modified version of contraction principle. Further, our prime aim is to present various Ulam-Hyers stability and $\mathbb{E}_{\alpha}$-Ulam-Hyers stability results via successive approximation method.
\end{abstract}

2010 Mathematics Subject Classification. 26A33, 34A08, 34K20.

Key words and phrases. Implicit fractional differential equation, Modified version of contraction principle, Existence, Ulam- Hyers Stability, Successive approximations.

\section{Introduction}

Theory of Ulam stability is the outcome of the question raised by S. M. Ulam in 1940 (See [1]). This theory is concerned with different kinds of equations such as: functional, integral, differential, difference etc. Hence it is a vast field of research and one of the most important growing subject in the area of mathematical analysis.

Motivation and basic theoretical development for the research related to Ulam-Hyers stability and Ulam-Hyers-Rassias stability problems to various forms of ordinary differential and integral equations of integer orders can be found in $[2,3,4,5,6,7,8,9,10]$ and the references given therein.

Interesting results have been obtained pertaining to different kinds of Ulam-Hyers stability for ordinary fractional differential and fractional integral equations with and without delay.

Received June 08, 2016 - Accepted November 24, 2016.

(c) The Author(s) 2016. This article is published with open access by Sidi Mohamed Ben Abdallah University.

${ }^{a}$ Department of Mathematics, Shivaji University, Kolhapur-416 004, Maharashtra, India.

${ }^{b}$ Raje Ramrao Mahavidyalaya, Jath, Sangli-416404, Maharashatra, India.

e-mail:kdkucche@gmail.com

e-mail: sutar.sagar007@gmail.com 
We mention here few recent works by Wang et al. [11, 12, 13, 14], Eghbali et al.[15] and Wei et al.[16]; also see the references cited therein.

Recently, Benchora et al. [17, 18, 19] obtained existence and various kinds of Ulam-Hyers stabilities for nonlinear implicit fractional differential equations (NIFDEs) involving Caputo fractional derivative with initial and boundary conditions. Kucche et al. [20, 21] have studied existence, uniqueness, continuous dependence and other properties of solutions for NIFDEs .

Here we consider the NIFDEs of the form:

$$
\begin{aligned}
{ }^{c} D^{\alpha} x(t) & =f\left(t, x(t),{ }^{c} D^{\alpha} x(t)\right), t \in[0, b], b>0, \text { for some } \alpha, m-1<\alpha \leq m \in \mathbb{N}, \\
x^{(k)}(0) & =x_{k} \in \mathbb{R}^{n}, \quad k=0,1, \cdots, m-1,
\end{aligned}
$$

where $f:[0, b] \times \mathbb{R}^{n} \times \mathbb{R}^{n} \rightarrow \mathbb{R}^{n}$ is a nonlinear continuous function, $x:[0, b] \rightarrow \mathbb{R}^{n}$ and ${ }^{c} D^{\alpha}$ denotes the Caputo fractional derivative of order $\alpha$ with lower terminal 0 .

In the present paper, we obtain existence and uniqueness of solutions for NIFDEs (1)-(2) using modified version of contraction principle. Taking motivation from $[6,10]$ we present the Ulam-Hyers stability and $\mathbb{E}_{\alpha^{-}}$-Ulam-Hyers stability results for NIFDE (1) by successive approximation method.

We remark that Ulam-Hyers stabilities for NIFDEs have been studied by Benchohra and Lazreg [18] using fixed point approach. Our attempt is here to establish Ulam-Hyers stability and $\mathbb{E}_{\alpha}$-Ulam-Hyers stability results for NIFDE (1) with initial conditions by virtue of successive approximation method.

\section{Preliminaries}

Consider the real space $\mathbb{R}^{n}$ with the norm $\|\cdot\|$ and denote by $B=C^{m}\left([0, b], \mathbb{R}^{n}\right)$-the Banach space all functions from $[0, b]$ into $\mathbb{R}^{n}$ having $m^{t h}$ order continuous derivatives endowed with supremum norm $\|\cdot\|_{B}$.

Here we give some basic definitions and the results $[22,23,24,25]$ which are required throughout this paper.

Definition 2.1. Let $g \in C[0, b]$ and $\alpha \geq 0$ then Riemann-Liouville fractional integral of order $\alpha$ of a function $f$ is defined as

$$
I^{\alpha} g(t)=\frac{1}{\Gamma(\alpha)} \int_{0}^{t}(t-s)^{\alpha-1} g(s) d s
$$

provided the integral exists. Note that $I^{0} g(t)=g(t)$.

Definition 2.2. Let $m-1<\alpha \leq m \in \mathbb{N}$. Then Caputo fractional derivative of a function $g \in C^{m}[0, b]$ is defined as

$$
{ }^{c} D^{\alpha} g(t)=\frac{1}{\Gamma(m-\alpha)} \int_{0}^{t}(t-s)^{m-\alpha-1} g^{(m)}(s) d s .
$$

Lemma 2.1. Let $m-1<\alpha \leq m \in \mathbb{N}$ and $g \in C^{m}[0, b]$. Then

$$
I^{\alpha}\left[{ }^{c} D^{\alpha} g(t)\right]=g(t)-\sum_{k=0}^{m-1} \frac{g^{(k)}(0)}{\Gamma(k+1)} t^{k} .
$$


Lemma 2.2. Let $g(t)=t^{\delta}$, where $\delta \geq 0$ and let $m-1<\alpha \leq m, m \in \mathbb{N}$, then

$$
{ }^{c} D^{\alpha} g(t)= \begin{cases}0 & \text { if } \delta \in\{0,1, \cdots, m-1\}, \\ \frac{\Gamma(1+\delta)}{\Gamma(1+\delta-\alpha)} t^{\delta-\alpha} & \text { if } \delta \in \mathbb{N}, \delta \geq m \text { or } \delta \notin \mathbb{N}, \delta>m-1 .\end{cases}
$$

Definition 2.3. Let $\gamma>0$, then one parameter Mittag-Leffler function of order $\gamma>0$ is defined by

$$
E_{\gamma}(z)=\sum_{k=0}^{\infty} \frac{z^{k}}{\Gamma(\gamma k+1)}
$$

We need the following results in our analysis.

Lemma 2.3. [26] For all $\mu>0$ and $\nu>-1$,

$$
\int_{0}^{t}(t-s)^{\mu-1} s^{\nu} d s=t^{\mu+\nu} \frac{\Gamma(\mu) \Gamma(\nu+1)}{\Gamma(\mu+\nu+1)} .
$$

In order to derive our result, we need the following generalized singular Gronwall inequality introduced by Ye et al.[27]

Lemma 2.4. [27] Suppose $\beta>0, \tilde{a}(t)$ is a nonnegative function locally integrable on $0 \leq$ $t<b, b \leq \infty$ and $\tilde{g}(t)$ is nonnegative, nondecreasing continuous functions defined and $\tilde{g}(t) \leq M, t \in(0, b]$, and suppose $y(t)$ is nonnegative and locally integrable on $(0, b]$ with

$$
y(t) \leq \tilde{a}(t)+\tilde{g}(t) \int_{0}^{t}(t-s)^{\beta-1} y(s) d s, t \in(0, b] .
$$

Then

$$
y(t) \leq \tilde{a}(t)+\int_{0}^{t}\left[\sum_{n=1}^{\infty} \frac{(\tilde{g}(t) \Gamma(\beta))^{n}}{\Gamma(n \beta)}(t-s)^{n \beta-1} \tilde{a}(s)\right] d s, \quad t \in(0, b] .
$$

Remark 2.1. Under the hypothesis of Lemma 2.4, let $\tilde{a}(t)$ be a nondecreasing function on $[0, b)$. Then we have

$$
y(t) \leq \tilde{a}(t) E_{\beta}\left(\tilde{g}(t) \Gamma(\beta) t^{\beta}\right) .
$$

To prove the existence and uniqueness results for (1)-(2), we use the modified version of contraction principle given below.

Lemma 2.5 ( [28], Modified version of contraction principle). Let $X$ be a Banach space and let $D$ be an operator which maps the element of $X$ into itself for which $D^{r}$ is a contraction, where $r$ is a positive integer then $D$ has a unique fixed point.

Definition 2.4. A function $x \in B$ is said to be a solution of (1)-(2) if $x$ satisfies the equation ${ }^{c} D^{\alpha} x(t)=f\left(t, x(t),{ }^{c} D^{\alpha} x(t)\right)$ on $[0, b]$, and also satisfies the initial conditions $x^{(k)}(0)=$ $x_{k}, k=0,1, \cdots, m-1$, where $m-1<\alpha \leq m \in \mathbb{N}$.

\section{Existence and uniqueness}

In the following lemma we obtain an equivalent fractional integral equation to the problem (1)-(2) 
Lemma 3.1. . Let $f:[0, b] \times \mathbb{R}^{n} \times \mathbb{R}^{n} \rightarrow \mathbb{R}^{n}$ be a continuous function. Then the NIFDEs (1)-(2) is equivalent to the fractional integral equation

$$
x(t)=\sum_{k=0}^{m-1} \frac{x_{k}}{\Gamma(k+1)} t^{k}+\frac{1}{\Gamma(\alpha)} \int_{0}^{t}(t-s)^{\alpha-1} p(s) d s, t \in[0, b],
$$

where $p \in B$ satisfies the functional equation

$$
p(t)=f\left(t, \sum_{k=0}^{m-1} \frac{x_{k}}{\Gamma(k+1)} t^{k}+I^{\alpha} p(t), p(t)\right), t \in[0, b] .
$$

Proof. Let $x:[0, b] \rightarrow \mathbb{R}^{n}$ in $B$ is a solution of NIFDE (1)-(2). Put

$$
{ }^{c} D^{\alpha} x(t)=p(t), t \in[0, b], m-1<\alpha \leq m(m \in \mathbb{N}),
$$

where $p \in B$. Operating $I^{\alpha}$ on both side, in the view of Lemma 2.1, we obtain

$$
x(t)=\sum_{k=0}^{m-1} \frac{x_{k}}{\Gamma(k+1)} t^{k}+I^{\alpha} p(t), t \in[0, b] .
$$

Thus equation(1) becomes

$$
\begin{aligned}
p(t) & =f(t, x(t), p(t)) \\
& =f\left(t, \sum_{k=0}^{m-1} \frac{x_{k}}{\Gamma(k+1)} t^{k}+I^{\alpha} p(t), p(t)\right), t \in[0, b] .
\end{aligned}
$$

Hence $x$ is solution of fractional integral equations (3), here $p \in B$ satisfies (4).

Conversely let $x:[0, b] \rightarrow \mathbb{R}^{n}$ in $B$ satisfies the integral equation (3) where $p \in B$ satisfies (4). Then equation (3) can be written as

$$
x(t)=\sum_{k=0}^{m-1} \frac{x_{k}}{\Gamma(k+1)} t^{k}+I^{\alpha} p(t), t \in[0, b] .
$$

Using Lemma 2.2 and the continuity of $p$, an application of the operator ${ }^{c} D^{\alpha}$ on both sides of the above equation, gives

$$
{ }^{c} D^{\alpha} x(t)=\sum_{k=0}^{m-1} \frac{x_{k}}{\Gamma(k+1)}{ }^{c} D^{\alpha}\left\{t^{k}\right\}+{ }^{c} D^{\alpha} I^{\alpha} p(t)=p(t), t \in[0, b] .
$$

But $p$ satisfies (4). Thus using (5) and (6) in (4), we obtain

$$
{ }^{c} D^{\alpha} x(t)=f\left(t, x(t),{ }^{c} D^{\alpha} x(t)\right), \quad t \in[0, b] .
$$

Further from (3), one can verify that

$$
x^{(k)}(0)=x_{k},(k=0,1, \cdots, m-1) .
$$

In the next theorem we prove the existence and uniqueness results for (1)-(2) by using modified version of contraction principle given in Lemma 2.5.

Theorem 3.1. (Existence and uniqueness) Let $f:[0, b] \times \mathbb{R}^{n} \times \mathbb{R}^{n} \rightarrow \mathbb{R}^{n}$ be a continuous function that satisfies the Lipschitz type condition: 
(H1) there exist constants $M>0$ and $0<N<1$ such that

$$
\|f(t, x, y)-f(t, \bar{x}, \bar{y})\| \leq M\|x-\bar{x}\|+N\|y-\bar{y}\|, t \in[0, b] ; x, \bar{x}, y, \bar{y} \in \mathbb{R}^{n} .
$$

Then the initial-value problem (1)-(2) has a unique solution $x:[0, b] \rightarrow \mathbb{R}^{n}$.

Proof. In the light of Lemma 3.1, we write the problem (1)-(2) as a fixed point problem.

Consider the operator $F: B \rightarrow B$ defined by

$$
F x(t)=\sum_{k=0}^{m-1} \frac{x_{k}}{\Gamma(k+1)} t^{k}+\frac{1}{\Gamma(\alpha)} \int_{0}^{t}(t-s)^{\alpha-1} p(s) d s, t \in[0, b],
$$

where $p \in B$ satisfies the functional equation

$$
p(t)=f(t, x(t), p(t)), t \in[0, b] .
$$

Our aim is to prove that $F$ has a fixed point. By using mathematical induction, for any $x, z \in B$ and $t \in[0, b]$, we prove that

$$
\left\|F^{j} x(t)-F^{j} z(t)\right\| \leq \frac{\left(\theta t^{\alpha}\right)^{j}}{\Gamma(j \alpha+1)}\|x-z\|_{B}, \forall j \in \mathbb{N},
$$

where $\theta=\left(\frac{M}{1-N}\right)>0$.

Let any $x, z \in B$ and $t \in[0, b]$. Then by definition of operator $F$ we have,

$$
\|F x(t)-F z(t)\| \leq \frac{1}{\Gamma(\alpha)} \int_{0}^{t}(t-s)^{\alpha-1}\|p(s)-q(s)\| d s,
$$

where $p, q \in B$ satisfies the functional equations

$$
p(t)=f(t, x(t), p(t)) \text { and } q(t)=f(t, z(t), q(t)), t \in[0, b] .
$$

By assumption (H1), for any $t \in[0, b]$, we have

$$
\begin{aligned}
\|p(t)-q(t)\| & =\|f(t, x(t), p(t))-f(t, z(t), q(t))\| \\
& \leq M\|x(t)-z(t)\|+N\|p(t)-q(t)\| .
\end{aligned}
$$

Therefore

$$
\|p(t)-q(t)\| \leq \theta\|x(t)-z(t)\|, t \in[0, b] .
$$

Using the above estimation in (8), we obtain

$$
\begin{aligned}
\|F x(t)-F z(t)\| & \leq \frac{\theta}{\Gamma(\alpha)} \int_{0}^{t}(t-s)^{\alpha-1}\|x(s)-z(s)\| d s \\
& \leq \frac{\theta}{\Gamma(\alpha)}\left(\int_{0}^{t}(t-s)^{\alpha-1} d s\right)\|x-z\|_{B} .
\end{aligned}
$$

Therefore,

$$
\|F x(t)-F z(t)\| \leq \frac{\theta t^{\alpha}}{\Gamma(\alpha+1)}\|x-z\|_{B}, t \in[0, b]
$$

Thus the inequality (7) holds for $j=1$. Let us suppose that (7) holds for $j=r \in \mathbb{N}$, that is,

$$
\left\|F^{r} x(t)-F^{r} z(t)\right\| \leq \frac{\left(\theta t^{\alpha}\right)^{r}}{\Gamma(r \alpha+1)}\|x-z\|_{B}, t \in[0, b] .
$$


We prove that (7) holds for $j=r+1$. Again, by definition of operator $F$, we have

$$
\begin{aligned}
\left\|\left(F^{r+1}\right) x(t)-\left(F^{r+1}\right) z(t)\right\| & =\left\|F\left(F^{r} x(t)\right)-F\left(F^{r} z(t)\right)\right\| \\
& \leq \frac{\theta}{\Gamma(\alpha)} \int_{0}^{t}(t-s)^{\alpha-1}\|h(s)-g(s)\| d s, t \in[0, b],
\end{aligned}
$$

where $h, g \in B$ are such that

$$
h(t)=f\left(t, F^{r} x(t), h(t)\right) \text { and } g(t)=f\left(t, F^{r} z(t), g(t)\right), t \in[0, b] .
$$

By assumption (H1) we have

$$
\|h(t)-g(t)\| \leq \theta\left\|F^{r} x(t)-F^{r} z(t)\right\|, t \in[0, b] .
$$

Hence we get,

$$
\left\|\left(F^{r+1}\right) x(t)-\left(F^{r+1}\right) z(t)\right\| \leq \frac{\theta}{\Gamma(\alpha)} \int_{0}^{t}(t-s)^{\alpha-1}\left\|F^{r} x(s)-F^{r} z(s)\right\| d s, t \in[0, b] .
$$

Using (9) and the Lemma 2.3 in the above inequality, we obtain

$$
\begin{aligned}
\left\|\left(F^{r+1}\right) x(t)-\left(F^{r+1}\right) z(t)\right\| & \leq \frac{\theta}{\Gamma(\alpha)} \int_{0}^{t}(t-s)^{\alpha-1} \frac{\left(\theta s^{\alpha}\right)^{r}}{\Gamma(r \alpha+1)}\|x-z\|_{B} d s \\
& \leq \frac{\theta^{r+1}}{\Gamma(\alpha) \Gamma(r \alpha+1)}\left(\int_{0}^{t}(t-s)^{\alpha-1} s^{r \alpha} d s\right)\|x-z\|_{B} \\
& =\frac{\theta^{r+1}}{\Gamma(\alpha) \Gamma(r \alpha+1)} t^{\alpha(r+1)} \frac{\Gamma(\alpha) \Gamma(r \alpha+1)}{\Gamma((r+1) \alpha+1)}\|x-z\|_{B} .
\end{aligned}
$$

Therefore,

$$
\left\|\left(F^{r+1}\right) x(t)-\left(F^{r+1}\right) z(t)\right\| \leq \frac{\left(\theta t^{\alpha}\right)^{r+1}}{\Gamma((r+1) \alpha+1)}\|x-z\|_{B}, t \in[0, b] .
$$

We have proved that the inequality (7) holds for $j=r+1$. By principle of mathematical induction the proof of the inequality (7) is completed.

From the inequality (7) we have

$$
\left\|F^{j} x-F^{j} z\right\|_{B}=\sup _{t \in[0, b]}\left\|F^{j} x(t)-F^{j} z(t)\right\| \leq \frac{\left(\theta b^{\alpha}\right)^{j}}{\Gamma(j \alpha+1)}\|x-z\|_{B} .
$$

By definition of one parameter Mittag-Leffler function, we have

$$
E_{\alpha}\left(\theta b^{\alpha}\right)=\sum_{j=0}^{\infty} \frac{\left(\theta b^{\alpha}\right)^{j}}{\Gamma(j \alpha+1)}
$$

Note that $\frac{\left(\theta b^{\alpha}\right)^{j}}{\Gamma(j \alpha+1)}$ is the $j^{\text {th }}$ term of the convergent series of nonnegative real numbers, hence we must have

$$
\lim _{j \rightarrow \infty} \frac{\left(\theta b^{\alpha}\right)^{j}}{\Gamma(j \alpha+1)}=0
$$

Thus we can choose $j \in \mathbb{N}$ such that $\frac{\left(\theta b^{\alpha}\right)^{j}}{\Gamma(j \alpha+1)}<1$ so that $F^{j}$ is a contraction. Therefore by modified version of contraction principle, $F$ has a unique fixed point $x:[0, b] \rightarrow \mathbb{R}^{n}$ in $B$, which is the unique solution of the initial value problem (1)-(2). 


\section{Ulam-Hyers Stability of NIFDE}

To study the stability results we use following definitions adopted in $[12,18]$.

Definition 4.1. We say that equation (1) has Ulam-Hyers stability if there exists a real number $K_{f}>0$ such that for each $\epsilon>0$, if $y:[0, b] \rightarrow \mathbb{R}^{n}$ in $B$ satisfies

$$
\left\|{ }^{c} D^{\alpha} y(t)-f\left(t, y(t),{ }^{c} D^{\alpha} y(t)\right)\right\| \leq \epsilon, t \in[0, b],
$$

then there exists a solution $x:[0, b] \rightarrow \mathbb{R}^{n}$ of equation (1) in $B$ with

$$
\|y(t)-x(t)\| \leq \epsilon K_{f}, t \in[0, b] .
$$

Definition 4.2. We say that equation (1) has generalized Ulam-Hyers stability if there exists $\psi \in C\left(\mathbb{R}_{+}, \mathbb{R}_{+}\right), \psi(0)=0$ such that for each $\epsilon>0$, if $y:[0, b] \rightarrow \mathbb{R}^{n}$ in $B$ satisfies

$$
\left\|{ }^{c} D^{\alpha} y(t)-f\left(t, y(t),{ }^{c} D^{\alpha} y(t)\right)\right\| \leq \epsilon, t \in[0, b],
$$

then there exists a solution $x:[0, b] \rightarrow \mathbb{R}^{n}$ of equation (1) in $B$ with

$$
\|y(t)-x(t)\| \leq \psi(\epsilon), t \in[0, b] .
$$

Definition 4.3. We say that equation (1) has Ulam-Hyers-Rassias stability with respect to with respect to $\eta \in C\left([0, b], \mathbb{R}_{+}\right)$if there exists a real number $K_{f}>0$ such that for each $\epsilon>0$, if $y:[0, b] \rightarrow \mathbb{R}^{n}$ in $B$ satisfies

$$
\left\|{ }^{c} D^{\alpha} y(t)-f\left(t, y(t),{ }^{c} D^{\alpha} y(t)\right)\right\| \leq \epsilon \eta(t), t \in[0, b],
$$

then there exists a solution $x:[0, b] \rightarrow \mathbb{R}^{n}$ of equation (1) in $B$ with

$$
\|y(t)-x(t)\| \leq K_{f} \in \eta(t), t \in[0, b] .
$$

Definition 4.4. We say that equation (1) has generalized Ulam-Hyers-Rassias stability with respect to with respect to $\eta \in C\left([0, b], \mathbb{R}_{+}\right)$if there exists a real number $K_{f, \eta}>0$ such that if $y:[0, b] \rightarrow \mathbb{R}^{n}$ in $B$ satisfies

$$
\left\|{ }^{c} D^{\alpha} y(t)-f\left(t, y(t),{ }^{c} D^{\alpha} y(t)\right)\right\| \leq \eta(t), t \in[0, b],
$$

then there exists a solution $x:[0, b] \rightarrow \mathbb{R}^{n}$ of equation (1) in $B$ with

$$
\|y(t)-x(t)\| \leq K_{f, \eta} \eta(t), t \in[0, b] .
$$

In the following theorems by method of successive approximation we prove Ulam-Hyers type stability results for equation (1).

Theorem 4.1. Let $f:[0, b] \times \mathbb{R}^{n} \times \mathbb{R}^{n} \rightarrow \mathbb{R}^{n}$ be a continuous function that satisfies the Lipschitz type condition (H1). For every $\epsilon>0$, if $y:[0, b] \rightarrow \mathbb{R}^{n}$ in $B$ satisfies

$$
\left\|{ }^{c} D^{\alpha} y(t)-f\left(t, y(t),{ }^{c} D^{\alpha} y(t)\right)\right\| \leq \epsilon, t \in[0, b],
$$

then there exists unique solution $x:[0, b] \rightarrow \mathbb{R}^{n}$ in $B$ of $\operatorname{NIFDE}(1)$ with $x^{(k)}(0)=y^{(k)}(0), k=$ $0,1,2, \ldots, m-1$, that satisfies

$$
\|y(t)-x(t)\| \leq\left(\frac{E_{\alpha}\left(\theta b^{\alpha}\right)-1}{\theta}\right) \epsilon, t \in[0, b],
$$

where $\theta=\frac{M}{(1-N)}>0$. 
Proof. For every $\epsilon>0$, let $y:[0, b] \rightarrow \mathbb{R}^{n}$ in $B$ satisfies

$$
\left\|{ }^{c} D^{\alpha} y(t)-f\left(t, y(t),{ }^{c} D^{\alpha} y(t)\right)\right\| \leq \epsilon, t \in[0, b],
$$

then there exists a function $\sigma_{y} \in B$ (depending on $y$ ) such that,

$$
\left\|\sigma_{y}(t)\right\| \leq \epsilon, t \in[0, b]
$$

and

$$
{ }^{c} D^{\alpha} y(t)=f\left(t, y(t),{ }^{c} D^{\alpha} y(t)\right)+\sigma_{y}(t), t \in[0, b]
$$

In the view of Lemma 3.1, $y$ satisfies the fractional integral equation

$$
y(t)=\sum_{k=0}^{m-1} \frac{y^{(k)}(0)}{\Gamma(k+1)} t^{k}+\frac{1}{\Gamma(\alpha)} \int_{0}^{t}(t-s)^{\alpha-1} p^{0}(s) d s+\frac{1}{\Gamma(\alpha)} \int_{0}^{t}(t-s)^{\alpha-1} \sigma_{y}(s) d s, t \in[0, b],
$$

where $p^{0} \in B$ is such that

$$
p^{0}(t)=f\left(t, y(t), p^{0}(t)\right), t \in[0, b] .
$$

Define,

$$
x^{0}(t)=y(t), t \in[0, b],
$$

and consider the sequence $\left\{x^{j}\right\} \subseteq B$ defined by ,

$$
x^{j}(t)=\sum_{k=0}^{m-1} \frac{y^{(k)}(0)}{\Gamma(k+1)} t^{k}+\frac{1}{\Gamma(\alpha)} \int_{0}^{t}(t-s)^{\alpha-1} p^{j-1}(s) d s, t \in[0, b], j \in \mathbb{N},
$$

where $p^{j-1} \in B(j \in \mathbb{N})$ is such that:

$$
p^{j-1}(t)=f\left(t, x^{j-1}(t), p^{j-1}(t)\right), t \in[0, b] .
$$

By principle of mathematical induction, we prove that

$$
\left\|x^{j}(t)-x^{j-1}(t)\right\| \leq \frac{\epsilon}{\theta} \frac{\left(\theta t^{\alpha}\right)^{j}}{\Gamma(j \alpha+1)}, j \in \mathbb{N}, t \in[0, b] .
$$

First we prove the inequality (13) for $\mathrm{j}=1$. Using the definition of successive approximations, for any $t \in[0, b]$, we have

$$
\begin{aligned}
\left\|x^{1}(t)-x^{0}(t)\right\| & =\left\|\sum_{k=0}^{m-1} \frac{y^{(k)}(0)}{\Gamma(k+1)} t^{k}+\frac{1}{\Gamma(\alpha)} \int_{0}^{t}(t-s)^{\alpha-1} p^{0}(s) d s-y(t)\right\| \\
& =\left\|\sum_{k=0}^{m-1} \frac{y^{(k)}(0)}{\Gamma(k+1)} t^{k}+I^{\alpha} p^{0}(t)-\left(\sum_{k=0}^{m-1} \frac{y^{(k)}(0)}{\Gamma(k+1)} t^{k}+I^{\alpha} p^{0}(t)+I^{\alpha} \sigma_{y}(t)\right)\right\| \\
& =\left\|I^{\alpha} \sigma_{y}(t)\right\| \\
& \leq \frac{1}{\Gamma(\alpha)} \int_{0}^{t}(t-s)^{\alpha-1}\left\|\sigma_{y}(s)\right\| d s \\
& \leq \frac{\epsilon t^{\alpha}}{\Gamma(\alpha+1)} .
\end{aligned}
$$

Therefore

$$
\left\|x^{1}(t)-x^{0}(t)\right\| \leq \frac{\epsilon}{\theta} \frac{\theta t^{\alpha}}{\Gamma(\alpha+1)}, t \in[0, b]
$$


which proves the inequality (13) for $j=1$. Now, we assume the inequality (13) hold for $j=r \in \mathbb{N}$ and prove it for $j=r+1$. Using definition of successive approximations for any $t \in[0, b]$,

$$
\begin{aligned}
\left\|x^{r+1}(t)-x^{r}(t)\right\| & =\left\|\frac{1}{\Gamma(\alpha)} \int_{0}^{t}(t-s)^{\alpha-1}\left(p^{r}(s)-p^{r-1}(s)\right) d s\right\| \\
& \leq \frac{1}{\Gamma(\alpha)} \int_{0}^{t}(t-s)^{\alpha-1}\left\|p^{r}(s)-p^{r-1}(s)\right\| d s .
\end{aligned}
$$

Since $p^{j}(t)=f\left(t, x^{j}(t), p^{j}(t)\right), t \in[0, b]$, using the assumption $(H 1)$ we obtain,

$$
\begin{aligned}
\left\|p^{r}(t)-p^{r-1}(t)\right\| & =\left\|f\left(t, x^{r}(t), p^{r}(t)\right)-f\left(t, x^{r-1}(t), p^{r-1}(t)\right)\right\| \\
& \leq M\left\|x^{r}(t)-x^{r-1}(t)\right\|+N\left\|p^{r}(t)-p^{r-1}(t)\right\| .
\end{aligned}
$$

Therefore

$$
\left\|p^{r}(t)-p^{r-1}(t)\right\| \leq \theta\left\|x^{r}(t)-x^{r-1}(t)\right\|, t \in[0, b] .
$$

Using above estimate and the inequality (13) for $j=r$ in (14), we obtain

$$
\begin{aligned}
\left\|x^{r+1}(t)-x^{r}(t)\right\| & \leq \frac{\theta}{\Gamma(\alpha)} \int_{0}^{t}(t-s)^{\alpha-1}\left\|x^{r}(s)-x^{r-1}(s)\right\| d s \\
& \leq \frac{\theta}{\Gamma(\alpha)} \int_{0}^{t}(t-s)^{\alpha-1} \frac{\epsilon}{\theta} \frac{\left(\theta s^{\alpha}\right)^{r}}{\Gamma(r \alpha+1)} d s \\
& =\frac{\epsilon}{\Gamma(\alpha)} \frac{\theta^{r}}{\Gamma(r \alpha+1)} \int_{0}^{t}(t-s)^{\alpha-1} s^{r \alpha} d s .
\end{aligned}
$$

Using Lemma 2.3, we have

$$
\left\|x^{r+1}(t)-x^{r}(t)\right\| \leq \frac{\epsilon}{\Gamma(\alpha)} \frac{\theta^{r}}{\Gamma(r \alpha+1)} t^{(r+1) \alpha} \frac{\Gamma(\alpha) \Gamma(r \alpha+1)}{\Gamma((r+1) \alpha+1)} .
$$

Therefore

$$
\left\|x^{r+1}(t)-x^{r}(t)\right\| \leq \frac{\epsilon}{\theta} \frac{\left(\theta t^{\alpha}\right)^{r+1}}{\Gamma((r+1) \alpha+1)}, t \in[0, b] .
$$

which is the inequality (13) for $j=r+1$. Using principle of mathematical induction the proof of the inequality (13) is completed.

Note that from (13),

$$
\left\|x^{j}(t)-x^{j-1}(t)\right\| \leq \frac{\epsilon}{\theta} \frac{\left(\theta b^{\alpha}\right)^{j}}{\Gamma(j \alpha+1)} \text { for any } t \in[0, b] \text { and } j \in \mathbb{N} .
$$

Therefore for any $t \in[0, b]$ we can write,

$$
\sum_{j=1}^{\infty}\left\|x^{j}(t)-x^{j-1}(t)\right\| \leq \frac{\epsilon}{\theta} \sum_{j=1}^{\infty} \frac{\left(\theta b^{\alpha}\right)^{j}}{\Gamma(j \alpha+1)}=\frac{\epsilon}{\theta}\left(E_{\alpha}\left(\theta b^{\alpha}\right)-1\right) .
$$

Hence the series

$$
x^{0}(t)+\sum_{j=1}^{\infty}\left[x^{j}(t)-x^{j-1}(t)\right]
$$

converges absolutely and uniformly on $[0, b]$ with respect to the norm $\|\cdot\|$. Let 


$$
x(t)=x^{0}(t)+\sum_{j=1}^{\infty}\left[x^{j}(t)-x^{j-1}(t)\right], t \in[0, b] .
$$

Then

$$
x^{r}(t)=x^{0}(t)+\sum_{j=1}^{r}\left[x^{j}(t)-x^{j-1}(t)\right]
$$

is the $r^{\text {th }}$ partial sum of the series (16) we have

$$
\lim _{r \rightarrow \infty}\left\|x^{r}(t)-x(t)\right\|=0, \text { for all } t \in[0, b] .
$$

Since the convergence is uniform, $x \in B$. We prove that the limit function $x$ is a solution of

$$
x(t)=\sum_{k=0}^{m-1} \frac{y^{(k)}(0)}{\Gamma(k+1)} t^{k}+\frac{1}{\Gamma(\alpha)} \int_{0}^{t}(t-s)^{\alpha-1} p(s) d s, t \in[0, b],
$$

where $p \in B$ satisfies the functional equation

$$
p(t)=f(t, x(t), p(t)), t \in[0, b] .
$$

First we prove that $p^{r} \in B(r=0,1,2, \ldots)$ generated in (12) satisfies

$$
\lim _{r \rightarrow \infty}\left\|p^{r}(t)-p(t)\right\|=0 \text { for any } t \in[0, b] .
$$

By using assumption $(H 1)$, we obtain

$$
\begin{aligned}
\left\|p^{r}(t)-p(t)\right\| & =\left\|f\left(t, x^{r}(t), p^{r}(t)\right)-f(t, x(t), p(t))\right\| \\
& \leq M\left\|x^{r}(t)-x(t)\right\|+N\left\|p^{r}(t)-p(t)\right\| .
\end{aligned}
$$

This gives

$$
\left\|p^{r}(t)-p(t)\right\| \leq \theta\left\|x^{r}(t)-x(t)\right\|, t \in[0, b] .
$$

Proof of (18) is completed using (17) and (19). Next, by definition of successive approximations

$$
\begin{aligned}
& \left\|x(t)-\sum_{k=0}^{m-1} \frac{y^{(k)}(0)}{\Gamma(k+1)} t^{k}-\frac{1}{\Gamma(\alpha)} \int_{0}^{t}(t-s)^{\alpha-1} p(s) d s\right\| \\
& =\left\|x(t)-x^{j}(t)+\frac{1}{\Gamma(\alpha)} \int_{0}^{t}(t-s)^{\alpha-1} p^{j-1}(s) d s-\frac{1}{\Gamma(\alpha)} \int_{0}^{t}(t-s)^{\alpha-1} p(s) d s\right\| \\
& \leq\left\|x(t)-x^{j}(t)\right\|+\frac{1}{\Gamma(\alpha)} \int_{0}^{t}(t-s)^{\alpha-1}\left\|p^{j-1}(s)-p(s)\right\| d s .
\end{aligned}
$$

Taking limit as $j \rightarrow \infty$ and noting the fact that left hand side of above inequality is independent of $j$, we obtain

$$
\left\|x(t)-\sum_{k=0}^{m-1} \frac{y^{(k)}(0)}{\Gamma(k+1)} t^{k}-\frac{1}{\Gamma(\alpha)} \int_{0}^{t}(t-s)^{\alpha-1} p(s) d s\right\|=0, t \in[0, b] .
$$

Therefore,

$$
x(t)=\sum_{k=0}^{m-1} \frac{y^{(k)}(0)}{\Gamma(k+1)} t^{k}+\frac{1}{\Gamma(\alpha)} \int_{0}^{t}(t-s)^{\alpha-1} p(s) d s, t \in[0, b] .
$$


This implies that $x(t)$ is solution of (1) with initial condition $x^{(k)}(0)=y^{(k)}(0) \in \mathbb{R}^{n}, k=$ $0,1, \ldots, m-1$. Further, from (15) and (16), we have

$$
\|y(t)-x(t)\| \leq\left(\frac{E_{\alpha}\left(\theta b^{\alpha}\right)-1}{\theta}\right) \epsilon, t \in[0, b] .
$$

This proves that the equation (1) is Ulam-Hyers stable. Moreover, as $x^{(k)}(0)=y^{(k)}(0), k=$ $0,1, \ldots, m-1$ the equation (1) has Ulam-Hyers stability with the initial condition.

It remains to prove the uniqueness of $x(t)$. Assume $\tilde{x}(t)$ is another solution of (1) with the initial conditions $\tilde{x}^{(k)}(0)=y^{(k)}(0), k=0,1, \cdots, m-1$. Then

$$
\tilde{x}(t)=\sum_{k=0}^{m-1} \frac{y^{(k)}(0)}{\Gamma(k+1)} t^{k}+\frac{1}{\Gamma(\alpha)} \int_{0}^{t}(t-s)^{\alpha-1} \tilde{p}(s) d s, t \in[0, b],
$$

where $\tilde{p} \in B$ satisfies $\tilde{p}(t)=f(t, \tilde{x}(t), \tilde{p}(t))$. Hence we have

$$
\|x(t)-\tilde{x}(t)\| \leq \frac{1}{\Gamma(\alpha)} \int_{0}^{t}(t-s)^{\alpha-1}\|p(s)-\tilde{p}(s)\| d s, t \in[0, b]
$$

Using $(H 1)$ we obtain

Thus

$$
\|p(t)-\tilde{p}(t)\| \leq \theta\|x(t)-\tilde{x}(t)\|
$$

$$
\|x(t)-\tilde{x}(t)\| \leq \frac{\theta}{\Gamma(\alpha)} \int_{0}^{t}(t-s)^{\alpha-1}\|x(s)-\tilde{x}(s)\| d s, t \in[0, b]
$$

An application of Lemma 2.4 to above inequality with $u(t)=\|x(t)-\tilde{x}(t)\|$ and $a(t)=0$, we obtain

$$
\|x(t)-\tilde{x}(t)\|=0 \text { for all } t \in[0, b] .
$$

This completes the proof.

Remark 4.1. If we set, $\psi(\epsilon)=\left(\frac{E_{\alpha}\left(\theta b^{\alpha}\right)-1}{\theta}\right) \epsilon$ then $\psi(0)=0$ and the equation (1) is generalized Ulam-Hyers stable.

Theorem 4.2. Let $f:[0, b] \times \mathbb{R}^{n} \times \mathbb{R}^{n} \rightarrow \mathbb{R}^{n}$ be a continuous function that satisfies the Lipschitz type condition (H1). In addition suppose that

(H2) Let $\eta \in C\left([0, b], \mathbb{R}_{+}\right)$be a nondecreasing function. There exists a constant $K>0$ satisfying $0<K \theta<1$ and

$$
\left\|\frac{1}{\Gamma(\alpha)} \int_{0}^{t}(t-s)^{\alpha-1} \eta(s) d s\right\| \leq K \eta(t), t \in[0, b]
$$

where $\theta=\frac{M}{(1-N)}>0$.

Then for every $\epsilon>0$ and $y:[0, b] \rightarrow \mathbb{R}^{n}$ in $B$ satisfying inequality

$$
\left\|^{c} D^{\alpha} y(t)-f\left(t, y(t),{ }^{c} D^{\alpha} y(t)\right)\right\| \leq \epsilon \eta(t), t \in[0, b],
$$

there exists unique solution $x:[0, b] \rightarrow \mathbb{R}^{n}$ in $B$ of $\operatorname{NIFDE}(1)$ with $x^{(k)}(0)=y^{(k)}(0), k=$ $0,1,2, \ldots, m-1$, that satisfies

$$
\|y(t)-x(t)\| \leq \epsilon \frac{K}{(1-K \theta)} \eta(t), t \in[0, b],
$$


Proof. For every $\epsilon>0$, let $y:[0, b] \rightarrow \mathbb{R}^{n}$ in $B$ satisfies

$$
\left\|{ }^{c} D^{\alpha} y(t)-f\left(t, y(t),{ }^{c} D^{\alpha} y(t)\right)\right\| \leq \epsilon \eta(t), t \in[0, b] .
$$

Then there exists a function $\sigma_{y} \in B$ (depending on $y$ ) such that

$$
\left\|\sigma_{y}(t)\right\| \leq \epsilon \eta(t), t \in[0, b]
$$

and

$$
{ }^{c} D^{\alpha} y(t)=f\left(t, y(t),{ }^{c} D^{\alpha} y(t)\right)+\sigma_{y}(t), t \in[0, b]
$$

In the view of Lemma 3.1, $y$ satisfies the fractional integral equation

$$
y(t)=\sum_{k=0}^{m-1} \frac{y^{(k)}(0)}{\Gamma(k+1)} t^{k}+\frac{1}{\Gamma(\alpha)} \int_{0}^{t}(t-s)^{\alpha-1} p^{0}(s) d s+\frac{1}{\Gamma(\alpha)} \int_{0}^{t}(t-s)^{\alpha-1} \sigma_{y}(s) d s, t \in[0, b],
$$

where $p^{0} \in B$ is such that

$$
p^{0}(t)=f\left(t, y(t), p^{0}(t)\right), t \in[0, b] .
$$

Considering the sequence $(11)$ with $x^{0}(t)=y(t), t \in[0, b]$ defined in proof of theorem 4.5, we prove by principle of mathematical induction that

$$
\left\|x^{j}(t)-x^{j-1}(t)\right\| \leq \frac{\epsilon}{\theta}(K \theta)^{j} \eta(t), j \in \mathbb{N}, t \in[0, b] .
$$

First we prove the inequality (22) for $\mathrm{j}=1$. Using the definition of successive approximations, for any $t \in[0, b]$, we have

$$
\begin{aligned}
\left\|x^{1}(t)-x^{0}(t)\right\| & =\left\|x^{1}(t)-y(t)\right\| \\
& =\left\|I^{\alpha} \sigma_{y}(t)\right\| \\
& \leq \frac{1}{\Gamma(\alpha)} \int_{0}^{t}(t-s)^{\alpha-1}\left\|\sigma_{y}(s)\right\| d s \\
& \leq \frac{\epsilon}{\Gamma(\alpha)} \int_{0}^{t}(t-s)^{\alpha-1} \eta(s) d s \\
& =\epsilon\left\|\frac{1}{\Gamma(\alpha)} \int_{0}^{t}(t-s)^{\alpha-1} \eta(s) d s\right\| \\
& \leq \epsilon K \eta(t) .
\end{aligned}
$$

Therefore

$$
\left\|x^{1}(t)-x^{0}(t)\right\| \leq \frac{\epsilon}{\theta}(K \theta) \eta(t), t \in[0, b],
$$

which proves the inequality (22) for $j=1$. Assuming that the inequality (22) hold for $j=r \in \mathbb{N}$ and proceeding as in the proof of theorem 4.5, we obtain

$$
\begin{aligned}
\left\|x^{r+1}(t)-x^{r}(t)\right\| & \leq \frac{\theta}{\Gamma(\alpha)} \int_{0}^{t}(t-s)^{\alpha-1}\left\|x^{r}(s)-x^{r-1}(s)\right\| d s \\
& \leq \frac{\theta}{\Gamma(\alpha)} \int_{0}^{t}(t-s)^{\alpha-1} \frac{\epsilon}{\theta}(K \theta)^{r} \eta(s) d s \\
& =\frac{\epsilon}{\Gamma(\alpha)}(K \theta)^{r} \int_{0}^{t}(t-s)^{\alpha-1} \eta(s) d s \\
& =\epsilon(K \theta)^{r}\left\|\frac{1}{\Gamma(\alpha)} \int_{0}^{t}(t-s)^{\alpha-1} \eta(s) d s\right\|
\end{aligned}
$$




$$
\leq \epsilon(K \theta)^{r} K \eta(t)
$$

Therefore

$$
\left\|x^{r+1}(t)-x^{r}(t)\right\| \leq \frac{\epsilon}{\theta}(K \theta)^{r+1} \eta(t), t \in[0, b] .
$$

which is the inequality (22) for $j=r+1$. By the principle of mathematical induction the proof of inequality (22) is completed. Using the inequality (22) and the assumption $0<K \theta<1$ we have

$$
\sum_{j=1}^{\infty}\left\|x^{j}(t)-x^{j-1}(t)\right\| \leq \frac{\epsilon}{\theta}\left(\sum_{j=1}^{\infty}(\theta K)^{j}\right) \eta(t)=\frac{\epsilon}{\theta}\left(\sum_{j=0}^{\infty}(\theta K)^{j}-1\right) \eta(t) .
$$

Therefore

$$
\sum_{j=1}^{\infty}\left\|x^{j}(t)-x^{j-1}(t)\right\| \leq \frac{\epsilon}{\theta}\left(\frac{1}{1-K \theta}-1\right) \eta(t)=\epsilon \frac{K}{1-K \theta} \eta(t) .
$$

Since the $\eta(t)$ continuous on compact set $[0, b]$ it is bounded and from above inequality it follows that the series

$$
x^{0}(t)+\sum_{j=1}^{\infty}\left[x^{j}(t)-x^{j-1}(t)\right]
$$

converges absolutely and uniformly on $[0, b]$ with respect to the norm $\|\cdot\|$. Set

$$
x(t)=x^{0}(t)+\sum_{j=1}^{\infty}\left[x^{j}(t)-x^{j-1}(t)\right], t \in[0, b],
$$

and proceeding in similar fashion as in the proof of theorem 4.1 we obtain

$$
\|y(t)-x(t)\| \leq \epsilon\left(\frac{K}{1-K \theta}\right) \eta(t), t \in[0, b] .
$$

This completes the proof.

Remark 4.2. If we set, $\epsilon=1$ and $K_{f, \eta}=\frac{K}{1-K \theta}$ then it follows that equation (1) is generalized Ulam-Hyers-Rassias stable.

\section{5. $\mathbb{E}_{\alpha}$-Ulam-Hyers stability}

We consider the following definitions of $\mathbb{E}_{\alpha}$-Ulam-Hyers stabilities introduced by Wang and Li [13].

Definition 5.1. We say that equation (1) has $\mathbb{E}_{\alpha}$-Ulam-Hyers stability if there exists a real number $K_{f}>0$ such that for each $\epsilon>0$ and each $y:[0, b] \rightarrow \mathbb{R}^{n}$ in $B$ satisfying

$$
\left\|{ }^{c} D^{\alpha} y(t)-f\left(t, y(t),{ }^{c} D^{\alpha} y(t)\right)\right\| \leq \epsilon, t \in[0, b],
$$

there exists a solution $x:[0, b] \rightarrow \mathbb{R}^{n}$ of equation (1) in $B$ with

$$
\|y(t)-x(t)\| \leq K_{f} E_{\alpha}\left(\gamma_{f} t^{\alpha}\right) \epsilon, \gamma_{f} \geq 0, t \in[0, b] .
$$


Definition 5.2. We say that equation (1) has generalized $\mathbb{E}_{\alpha}$-Ulam-Hyers stability if there exists a function $\psi \in C\left(\mathbb{R}_{+}, \mathbb{R}_{+}\right), \psi(0)=0$, such that for each $\epsilon>0$ and each $y:[0, b] \rightarrow \mathbb{R}^{n}$ in $B$ satisfying

$$
\left\|{ }^{c} D^{\alpha} y(t)-f\left(t, y(t),{ }^{c} D^{\alpha} y(t)\right)\right\| \leq \epsilon, t \in[0, b],
$$

there exists a solution $x:[0, b] \rightarrow \mathbb{R}^{n}$ of equation (1) in $B$ with

$$
\|y(t)-x(t)\| \leq \psi(\epsilon) E_{\alpha}\left(\gamma_{f} t^{\alpha}\right), \gamma_{f} \geq 0, t \in[0, b] .
$$

Definition 5.3. We say that equation (1) has $\mathbb{E}_{\alpha}$-Ulam-Hyers-Rassias stability with respect to with respect to $\eta \in C\left([0, b], \mathbb{R}_{+}\right)$if there exists a real number $K_{\eta}>0$ such that for each $\epsilon>0$, if $y:[0, b] \rightarrow \mathbb{R}^{n}$ in $B$ satisfies

$$
\left\|{ }^{c} D^{\alpha} y(t)-f\left(t, y(t),{ }^{c} D^{\alpha} y(t)\right)\right\| \leq \epsilon \eta(t), t \in[0, b],
$$

then there exists a solution $x:[0, b] \rightarrow \mathbb{R}^{n}$ of equation (1) in $B$ with

$$
\|y(t)-x(t)\| \leq K_{\eta} E_{\alpha}\left(\gamma_{f} t^{\alpha}\right) \epsilon \eta(t), t \in[0, b] .
$$

Definition 5.4. We say that equation (1) has generalized $\mathbb{E}_{\alpha}$-Ulam-Hyers-Rassias stability with respect to with respect to $\eta \in C\left([0, b], \mathbb{R}_{+}\right)$if there exists a real number $K_{\eta}>0$ such that if $y:[0, b] \rightarrow \mathbb{R}^{n}$ in $B$ satisfies

$$
\left\|{ }^{c} D^{\alpha} y(t)-f\left(t, y(t),{ }^{c} D^{\alpha} y(t)\right)\right\| \leq \eta(t), t \in[0, b],
$$

then there exists a solution $x:[0, b] \rightarrow \mathbb{R}^{n}$ of equation (1) in $B$ with

$$
\|y(t)-x(t)\| \leq K_{\eta} E_{\alpha}\left(\gamma_{f} t^{\alpha}\right) \eta(t), t \in[0, b] .
$$

By the method of successive approximations we now prove the $\mathbb{E}_{\alpha}$-Ulam type stbility results for equation (1).

Theorem 5.1. Let $f:[0, b] \times \mathbb{R}^{n} \times \mathbb{R}^{n} \rightarrow \mathbb{R}^{n}$ be a continuous function that satisfies Lipschitz type condition (H1). For every $\epsilon>0$, if $y:[0, b] \rightarrow \mathbb{R}^{n}$ in $B$ satisfies

$$
\left\|{ }^{c} D^{\alpha} y(t)-f\left(t, y(t),{ }^{c} D^{\alpha} y(t)\right)\right\| \leq \epsilon, t \in[0, b],
$$

then there exists unique solution $x:[0, b] \rightarrow \mathbb{R}^{n}$ in $B$ of NIFDE (1) with $x^{k}(0)=y^{k}(0), k=$ $0,1,2, \ldots, m-1$, that satisfies

$$
\|y(t)-x(t)\| \leq \frac{1}{\theta} E_{\alpha}\left(\theta t^{\alpha}\right) \epsilon, t \in[0, b]
$$

Proof. Noting that $x^{0}(t)=y(t)$, we write from (13) and (16)

$$
\|y(t)-x(t)\| \leq \sum_{j=1}^{\infty}\left\|x^{j}(t)-x^{j-1}(t)\right\| \leq \sum_{j=0}^{\infty} \frac{\epsilon}{\theta} \frac{\left(\theta t^{\alpha}\right)^{j}}{\Gamma(j \alpha+1)} \leq \frac{\epsilon}{\theta} E_{\alpha}\left(\theta t^{\alpha}\right), t \in[0, b] .
$$

Showing that the the initial value problem (1) is $\mathbb{E}_{\alpha}$-Ulam-Hyers stable with initial conditions. 
Remark 5.1. Set, $\psi(\epsilon)=\frac{\epsilon}{\theta}$ then $\psi(0)=0$, and it follows that the equation (1) is generalized $\mathbb{E}_{\alpha}$-Ulam-Hyers stable.

Theorem 5.2. Let $f:[0, b] \times \mathbb{R}^{n} \times \mathbb{R}^{n} \rightarrow \mathbb{R}^{n}$ be a continuous function that satisfies the Lipschitz type condition (H1) and suppose that condition (H2) hold. Then for every $\epsilon>0$ and $y:[0, b] \rightarrow \mathbb{R}^{n}$ in $B$ satisfying inequality

$$
\left\|^{c} D^{\alpha} y(t)-f\left(t, y(t),{ }^{c} D^{\alpha} y(t)\right)\right\| \leq \epsilon \eta(t), t \in[0, b],
$$

there exists unique solution $x:[0, b] \rightarrow \mathbb{R}^{n}$ in $B$ of $\operatorname{NIFDE}(1)$ with $x^{(k)}(0)=y^{(k)}(0), k=$ $0,1,2, \ldots, m-1$, that satisfies

$$
\|y(t)-x(t)\| \leq \epsilon \frac{K^{2}+K}{(1-K \theta)} \eta(t) E_{\alpha}\left(t^{\alpha}\right), t \in[0, b] .
$$

Proof. Noting that $x^{0}(t)=y(t)$, we write from (22) and (24)

$$
\begin{aligned}
\|y(t)-x(t)\| & \leq \sum_{j=1}^{\infty}\left\|x^{j}(t)-x^{j-1}(t)\right\| \leq \sum_{j=1}^{\infty} \frac{\epsilon}{\theta}(K \theta)^{j} \eta(t) \\
& =\frac{\epsilon}{\theta} \frac{K \theta}{(1-K \theta)} \eta(t)=\epsilon \frac{K}{(1-K \theta)} \eta(t), t \in[0, b] .
\end{aligned}
$$

Operating $I^{\alpha}$ on both sides of above inequality and using assumption $\left(H_{2}\right)$ we get,

$$
I^{\alpha}\|y(t)-x(t)\| \leq \epsilon \frac{K}{(1-K \theta)} I^{\alpha} \eta(t) \leq \epsilon \frac{K^{2}}{(1-K \theta)} \eta(t), t \in[0, b] .
$$

Adding theses two inequalities we get,

$$
\|y(t)-x(t)\|+I^{\alpha}\|y(t)-x(t)\| \leq \epsilon \frac{K^{2}+K}{(1-K \theta)} \eta(t), t \in[0, b] .
$$

Hence,

$$
\|y(t)-x(t)\| \leq \epsilon \frac{K^{2}+K}{(1-K \theta)} \eta(t)-I^{\alpha}\|y(t)-x(t)\|, t \in[0, b] .
$$

Noting that $\|y(t)-x(t)\| \geq 0$, we write above inequality as,

$$
\begin{aligned}
\|y(t)-x(t)\| & \leq \epsilon \frac{K^{2}+K}{(1-K \theta)} \eta(t)+I^{\alpha}\|y(t)-x(t)\| \\
& =\epsilon \frac{K^{2}+K}{(1-K \theta)} \eta(t)+\frac{1}{\Gamma(\alpha)} \int_{0}^{t}(t-s)^{\alpha-1}\|y(s)-x(s)\| d s, t \in[0, b] .
\end{aligned}
$$

An application of Lemma 2.4 to above inequality with $u(t)=\|y(t)-x(t)\|, \widetilde{a}(t)=\epsilon \frac{K^{2}+K}{(1-K \theta)} \eta(t)$ and $\widetilde{g}(t)=\frac{1}{\Gamma(\alpha)}$ we obtain

$$
\|y(t)-x(t)\| \leq \epsilon \frac{K^{2}+K}{(1-K \theta)} \eta(t) E_{\alpha}\left(\frac{1}{\Gamma(\alpha)} \Gamma(\alpha) t^{\alpha}\right)=\epsilon \frac{K^{2}+K}{(1-K \theta)} \eta(t) E_{\alpha}\left(t^{\alpha}\right)
$$

Showing that the the initial value problem (1) is $\mathbb{E}_{\alpha^{-}}$Ulam-Hyers-Rassias stable with initial conditions.

Remark 5.2. Set, $\epsilon=1$ then $K_{f, \eta}=\frac{K^{2}+K}{(1-K \theta)}$, and it follows that the equation (1) is generalized $\mathbb{E}_{\alpha}$-Ulam-Hyers-Rassias stable. 


\section{Example}

In this section, we give an example in support of the results we obtained.

6.1. Example. Let $\mathbb{R}^{2}$ be the normed space with the norm

$$
\|x\|=\left|x_{1}\right|+\left|x_{2}\right|, x=\left(x_{1}, x_{2}\right) \in \mathbb{R}^{2} .
$$

Consider the NIFDEs of the form:

$$
\begin{aligned}
{ }^{c} D^{\frac{7}{2}} x(t) & =f\left(t, x(t),{ }^{c} D^{\frac{7}{2}} x(t)\right), t \in[0,1.5], \\
x^{(k)}(0) & =x_{k} \in \mathbb{R}^{2}, \quad k=0,1,2,3 .
\end{aligned}
$$

where $x:[0,1.5] \rightarrow \mathbb{R}^{2}$ and $f:[0,1.5] \times \mathbb{R}^{2} \times \mathbb{R}^{2} \rightarrow \mathbb{R}^{2}$ is a nonlinear function defined by

$$
\begin{aligned}
f\left(t, x(t),{ }^{c} D^{\frac{7}{2}} x(t)\right) & =f\left(t,\left(x_{1}(t), x_{2}(t)\right),\left({ }^{c} D^{\frac{7}{2}} x_{1}(t),{ }^{c} D^{\frac{7}{2}} x_{2}(t)\right)\right) \\
& =\left(\frac{\log \left(2+t^{2}\right)}{1+\left|x_{1}(t)\right|+\left|x_{2}(t)\right|}, \frac{\left|{ }^{c} D^{\frac{7}{2}} x_{1}(t)\right|+\left|{ }^{c} D^{\frac{7}{2}} x_{2}(t)\right|}{e^{t^{2}+1}\left(1+\left|{ }^{c} D^{\frac{7}{2}} x_{1}(t)\right|+\left|{ }^{c} D^{\frac{7}{2}} x_{2}(t)\right|\right)}\right), t \in[0,1.5] .
\end{aligned}
$$

For any $x=\left(x_{1}, x_{2}\right), y=\left(y_{1}, y_{2}\right), \bar{x}=\left(\bar{x}_{1}, \bar{x}_{2}\right), \bar{y}=\left(\bar{y}_{1}, \bar{y}_{2}\right) \in \mathbb{R}^{2}$, we have

$$
\begin{aligned}
& \|f(t, x, y)-f(t, \bar{x}, \bar{y})\| \\
& =\left\|f\left(t,\left(x_{1}, x_{2}\right),\left(y_{1}, y_{2}\right)\right)-f\left(t,\left(\bar{x}_{1}, \bar{x}_{2}\right),\left(\bar{y}_{1}(t), \bar{y}_{2}\right)\right)\right\| \\
& =\|\left(\frac{\log \left(2+t^{2}\right)}{1+\left|x_{1}\right|+\left|x_{2}\right|}, \frac{\left|y_{1}\right|+\left|y_{2}\right|}{e^{t^{2}+1}\left(1+\left|y_{1}\right|+\left|y_{2}\right|\right)}\right) \\
& \quad-\left(\frac{\log \left(2+t^{2}\right)}{1+\left|\bar{x}_{1}\right|+\left|\bar{x}_{2}\right|}, \frac{\left|\bar{y}_{1}\right|+\left|\bar{y}_{2}\right|}{e^{t^{2}+1}\left(1+\left|\bar{y}_{1}\right|+\left|\bar{y}_{2}\right|\right)}\right) \| \\
& =\|\left(\log \left(2+t^{2}\right)\left[\frac{1}{1+\left|x_{1}\right|+\left|x_{2}\right|}-\frac{1}{1+\left|\bar{x}_{1}\right|+\left|\bar{x}_{2}\right|}\right],\right. \\
& =\|\left(\log \left(2+t^{2}\right)\left[\frac{\left|\bar{x}_{1}\right|-\left|x_{1}\right|+\left|\bar{x}_{2}(t)\right|-\left|x_{2}\right|}{\left(1+\left|x_{1}\right|+\left|x_{2}\right|\right)\left(1+\left|\bar{x}_{1}\right|+\left|\bar{x}_{2}\right|\right)}\right],\right. \\
& \left.\quad \frac{1}{e^{t^{2}+1}}\left[\frac{\left|y_{1}\right|+\left|y_{2}\right|}{1+\left|y_{1}\right|+\left|y_{2}\right|}-\frac{\left|\bar{y}_{2}\right|}{1+\left|\bar{y}_{1}\right|+\left|\bar{y}_{2}\right|}\right]\right) \| \\
& \left.=\log \left(2+t^{2}\right)\left|\frac{\left|\bar{y}_{1}\right|+\left|y_{2}\right|-\left|\bar{y}_{2}\right|}{\left(1+\left|\bar{x}_{1}\right|-\left|x_{1}\right|+\left|\bar{x}_{2}\right|-\left|x_{2}\right|\right.}\right|\right) \mid \\
& \quad \frac{\left.1+\left|x_{2}\right|\right)\left(1+\left|\bar{x}_{1}\right|+\left|\bar{x}_{2}\right|\right) \mid}{e^{t^{2}+1}}\left[\frac{\left|y_{1}\right|-\left|\bar{y}_{1}\right|+\left|y_{2}\right|-\left|\bar{y}_{2}\right|}{\left(1+\left|y_{2}\right|\right)\left(1+\bar{y}_{1} \mid\right)\left(1+\left|\bar{y}_{1}\right|+\left|\bar{y}_{2}\right|\right)} \mid\right)
\end{aligned}
$$

Note that for any $a, b \geq 0$ we have

$$
1 \leq(1+a+b)
$$

Therefore

$$
\begin{aligned}
& \|f(t, x, y)-f(t, \bar{x}, \bar{y})\| \\
& \leq \log \left(2+t^{2}\right)\left|\left(\left|\bar{x}_{1}\right|-\left|x_{1}\right|+\left|\bar{x}_{2}\right|-\left|x_{2}\right|\right)\right|+\frac{1}{e^{t^{2}+1}}\left|\left(\left|y_{1}\right|-\left|\bar{y}_{1}\right|+\left|y_{2}\right|-\left|\bar{y}_{2}\right|\right)\right|
\end{aligned}
$$




$$
\begin{aligned}
& \leq \log \left(2+t^{2}\right)\|(\|\bar{x}\|-\|x\|)\|+\frac{1}{e^{t^{2}+1}}\|(\|y\|-\|\bar{y}\|)\| \\
& \leq \log (4.25)\|x-\bar{x}\|\left\|+\frac{1}{e}\right\| y-\bar{y} \| .
\end{aligned}
$$

Thus the function $f$ satisfies condition $(H 1)$ with $M=\log (4.25)>0$ and $0<N=\frac{1}{e}<1$. Hence by Theorem 3.2 equation (25)-(26) has unique solution on $[0,1.5]$.

Moreover, as shown in Theorem 4.1 , for every $\epsilon>0$ if $y:[0,1.5] \rightarrow \mathbb{R}^{2}$ satisfies

$$
\left\|{ }^{c} D^{\frac{7}{2}} y(t)-f\left(t, y(t),{ }^{c} D^{\frac{7}{2}} y(t)\right)\right\| \leq \epsilon, t \in[0,1.5],
$$

there exists unique solution $x:[0,1.5] \rightarrow \mathbb{R}^{2}$ such that

$$
\|y(t)-x(t)\| \leq\left(\frac{E_{\frac{7}{2}}\left(\theta(1.5)^{\frac{7}{2}}\right)-1}{\theta}\right) \epsilon \text { for all } t \in[0,1.5],
$$

where $\theta=\frac{M}{1-N}=\frac{\log 4.25}{\left(1-\frac{1}{e}\right)}=\frac{e \log 4.25}{(e-1)}$. Hence NIFDE (25) is Ulam-Hyers stable. Further, as discussed in Remark 4.1 NIFDE (25) is also generalized Ulam-Hyers stable.

Again, for every $\epsilon>0$ if $y:[0,1.5] \rightarrow \mathbb{R}^{2}$ satisfies (27) by Theorem 5.5 there exists unique solution $x:[0,1.5] \rightarrow \mathbb{R}^{2}$ such that

$$
\|y(t)-x(t)\| \leq \frac{1}{\theta} E_{\frac{7}{2}}\left(\theta(1.5)^{\frac{7}{2}}\right) \epsilon, t \in[0,1.5] .
$$

Thus NIFDE (25) is $\mathbb{E}_{\frac{7}{2}}$ - Ulam-Hyers stable and by Remark 5.1 it is generalized $\mathbb{E}_{\frac{7}{2}}$-UlamHyers stable.

Define the function $\eta(t)=C E_{\frac{7}{2}}\left(t^{\frac{7}{2}}\right)$, where $C$ is a constant. Then $\eta(t)$ is nondecreasing function is such that

$$
I^{\frac{7}{2}}(\eta(t))=I^{\frac{7}{2}}\left(C E_{\frac{7}{2}}\left(t^{\frac{7}{2}}\right)\right)=C I^{\frac{7}{2}}\left(E_{\frac{7}{2}}\left(t^{\frac{7}{2}}\right)\right) \leq C E_{\frac{7}{2}}\left(t^{\frac{7}{2}}\right)=\eta(t), t \in[0,1.5] .
$$

Thus the function $\eta(t)$ satisfies the condition (H2) with $K=1$. Further, $0<K \theta=\theta=$ $\frac{e \log 4.25}{(e-1)}<1$.

For $\epsilon>0$ and sufficiently large value of $C$, let $y:[0,1.5] \rightarrow \mathbb{R}^{2}$ satisfy

$$
\left\|^{c} D^{\frac{7}{2}} y(t)-f\left(t, y(t),{ }^{c} D^{\frac{7}{2}} y(t)\right)\right\| \leq \epsilon \eta(t), t \in[0,1.5] .
$$

Then by Theorem 4.2 , we get a solution $x:[0,1.5] \rightarrow \mathbb{R}^{2}$ of equation (25) satisfying,

$$
\|y(t)-x(t)\| \leq \epsilon \frac{1}{(1-\theta)} \eta(t), t \in[0,1.5] .
$$

and hence the equation (25) is Ulam-Hyers-Rassias stable. Also as mentioned in Remark 4.2 equation (25) is generalized Ulam-Hyers-Rassias stable.

For every $\epsilon>0$ if $y:[0,1.5] \rightarrow \mathbb{R}^{2}$ satisfies (28) by Theorem 5.2 there exists a solution $x:[0,1.5] \rightarrow \mathbb{R}^{2}$ such that

$$
\|y(t)-x(t)\| \leq \epsilon \frac{2}{(1-\theta)} \eta(t) E_{\frac{7}{2}}\left(t^{\frac{7}{2}}\right), t \in[0,1.5] .
$$

shows that the equation (25) is $\mathbb{E}_{\frac{7}{2}}$-Ulam-Hyers-Rassias stable and by Remark 5.2 equation (25) is a generalized $\mathbb{E}_{\frac{7}{2}}$-Ulam-Hyers-Rassias stable. 
Acknowledgments. We express our sincere gratitude to anonymous referees for their valuable comments and suggestions.

\section{References}

[1] S. M. Ulam, A collection of the mathematical problems, Interscience Publ., New York, 1960.

[2] J. Wang, M. Feckan,Y. Zhou, Ulam's type stability of impulsive ordinary differential equations, $J$. Math. Anal. Appl. 395 (2012), 258-264.

[3] S.M. Jung, J. Brzdek, Hyers-Ulam stability of the delay equation $y^{\prime}(t)=y(t-\tau)$, Abstr. Appl. Anal.(2010) 88 Article ID 372176,10 pages.

[4] S. M. Jung, A fixed point approach to the stability of linear differential $y^{\prime}=f(x, y)$, Bull. Malays. Math. Sci. Soc. 33 (2) (2010), 47-56.

[5] S.M. Jung, Hyers-Ulam stability of linear differential equations of first order, Appl. Math. Lett. 17 (2004), 1135-1140.

[6] M. Gachpazan, O. Baghani, Hyers-Ulam stability of nonlinear integral equation, Fixed Point Theory and Applications Volume (2010) Article ID 927640, 6 pages.

[7] D. Otrocol, V. Ilea, Ulam stability for a delay differential equation, Cent. Eur. J. Math. 11(7) ( 2013), 1296-1303.

[8] Y. Li, Y. Shen, Hyers-Ulam stability of linear differential equations of second order, Appl. Math. Lett. 23 (2010), 306-309.

[9] J. Huang, S. M. Jung,Y. Li, Hyers-Ulam stability of nonlinear differential equations, Bull,Korean Math.Soc. 43 (2006), 107-117.

[10] J. Huang, Y. Li, Hyers-Ulam stability of delay differential equations of first order, Math. Nachr.(2015), 1-7.

[11] J. Wang,L. Lv,Y. Zhou, New concepts and results in stability of fractional differential equations, Commun Nonlinear Sci Numer Simulat 17 (2012), 2530-2538.

[12] J. Wang, L. Lv, Y. Zhou, Ulam stability and data dependence for fractional differential equations with Caputo derivative, Qualit Th Diff Equat 63 (2011), 1-10.

[13] J. Wang, X. Li, $\mathbb{E}_{\alpha}$-Ulam type stability of fractional order ordinary differential equations, J. Appl. Math. Comput. 45 (2014), 449-459.

[14] J. Wang, Y. Zhanga, Ulam-Hyers-Mittag-Leffler stability of fractional-order delay differential equations, Optimization 64 (8) (2014), 1181-1190.

[15] N. Eghbali, V. Kalvandi, J. M. Rassias, A fixed point approach to the Mittag-Leffler-Hyers-Ulam stability of a fractional integral equation, Open Math. 14 (2016), 237-246.

[16] Wei Wei, Xuezhu Li, Xia Li, New stability results for fractional integral equations, Computers and Mathematics with Applications 64 (2012), 3468-3476.

[17] M. Benchohra, M. Said Souid, Integrable solutions for implicit fractional order differential equations, TJMM, 6 (2) (2014), 101-107.

[18] M. Benchohra, J. Lazreg, On stability for nonlinear implicit fractional differential equations, Le Matematiche Vol. LXX (2015) Fasc. II, 49-61.

[19] M. Benchohraa, S. Bouriaha, Existence and stability results for nonlinear boundary value problem for implicit differential equations of fractional order, Moroccan J. Pure and Appl. Anal., 1(1) (2015), $22-37$.

[20] K. D. Kucche, J. J. Nieto, V. Venktesh, Theory of nonlinear implicit fractional differential equations, Differ. Equ. Dyn. Syst. DOI 10.1007/s12591-016-0297-7.

[21] S.T.Sutar, K.D.Kucche, Global existence and uniqueness for implicit differential equations of arbitrary order, Fractional Differential Calculus 5(2)(2015), 199-208.

[22] K. S. Miller, B. Ross, An introduction to the fractional calculus and differential equations, John Wiley, New York, 1993.

[23] I. Podlubny, Fractional differential equations, Academic Press, San Diego, 1999. 
[24] A. A. Kilbas, H. M. Srivastava, J. J. Trujillo, Theory and applications of fractional differential equations, North-Holland Mathematics Studies, vol. 204, Elsevier Science B.V., Amsterdam, 2006. [25] K. Diethelm, The analysis of fractional differential equations, Lecture Notes in Mathematics, Springer-verlag; Berlin, Heidelberg, 2010.

[26] Michalski MW, Derivatives of noninteger order and their applications, Dissertationes Mathematicae.Polska Akademia Nauk.,Instytut Matematyczny, Warszawa;1993.

[27] H. Ye, J. Gao, Y. Ding, A generalized Gronwall inequality and its application to a fractional differential equation, J. Math. Anal. Appl., 328 (2007), 1075-1081.

[28] A. H. Siddiqi, Functional analysis with applications, Tata McGraw-Hill Publishing ltd , New Delhi, 1986. 